\title{
The neutron polaron as a constraint on nuclear density functionals
}

\author{
M. M. Forbes, ${ }^{1,2,3}$ A. Gezerlis, ${ }^{4,5,6, \text { 冈 K. Hebeler, }}{ }^{5,6,7}$ T. Lesinski, ${ }^{1,2,8,9}$ and A. Schwenk ${ }^{6,5}$ \\ ${ }^{1}$ Institute for Nuclear Theory, University of Washington, Seattle, Washington 98195-1560, USA \\ ${ }^{2}$ Department of Physics, University of Washington, Seattle, Washington 98195-1560, USA \\ ${ }^{3}$ Department of Physics and Astronomy, Washington State University, Pullman, Washington 99164-2814 \\ ${ }^{4}$ Department of Physics, University of Guelph, Guelph, Ontario N1G 2W1, Canada \\ ${ }^{5}$ Institut für Kernphysik, Technische Universität Darmstadt, 64289 Darmstadt, Germany \\ ${ }^{6}$ ExtreMe Matter Institute EMMI, GSI Helmholtzzentrum für Schwerionenforschung GmbH, 64291 Darmstadt, Germany \\ ${ }^{7}$ Department of Physics, The Ohio State University, Columbus, OH 43210, USA \\ ${ }^{8}$ University of Warsaw, Institute of Theoretical Physics, ul. Hoża 69, 00-681 Warsaw, Poland \\ ${ }^{9}$ CEA Saclay, IRFU/Service de Physique Nucléaire, F-91191 Gif-sur-Yvette, France
}

\begin{abstract}
We study the energy of an impurity (polaron) that interacts strongly in a sea of fermions when the effective range of the impurity-fermion interaction becomes important, thereby mapping the Fermi polaron of condensed matter physics and ultracold atoms to strongly interacting neutrons. We present Quantum Monte Carlo results for this neutron polaron, and compare these with effective field theory calculations that also include contributions beyond the effective range. We show that stateof-the-art nuclear density functionals vary substantially and generally underestimate the neutron polaron energy. Our results thus provide constraints for adjusting the time-odd components of nuclear density functionals to better characterize polarized systems.
\end{abstract}

PACS numbers: 03.75.Ss, 05.30.Fk, 21.65.Cd, 21.60.Jz

Energy-density functionals are the only method available to study heavy nuclei and to globally describe the chart of nuclides [1, 2. Due to the need for a precise description of low-energy observables, parameters of these functionals are generally fit to properties of nuclei, including masses and radii. This empirical construction can therefore also benefit from additional input to constrain their properties in exotic, i.e., neutron-rich or spin-polarized systems. Examples of such pseudo-data include calculations of neutron matter [3 9 ] and neutron drops [10]. This approach has been successfully used to shape new functionals (see, e.g., Refs. [11 16]). In this work, we study the neutron polaron and use its energy as a constraint on nuclear density functionals.

The polaron was first introduced in condensed matter physics, and has recently been investigated in strongly interacting ultracold Fermi gases [17] - a system that has many similarities with the physics of low-density neutron matter (see, e.g., Refs. 3, 4). The Fermi polaron is an impurity interacting in a Fermi sea, realized in ultracold atoms and neutron matter as a spin-down fermion in a sea of $N_{\uparrow}$ spin-up fermions. The polaron energy $E_{\text {pol }}=$ $E_{N_{\uparrow}+1}-E_{N_{\uparrow}}$ is defined as the energy difference between the system with the polaron added and the $N_{\uparrow}$ (noninteracting) Fermi system. In the thermodynamic limit, this is equivalent to the spin-down chemical potential in the limit of high polarization, and therefore constrains the phase diagram of strongly interacting Fermi systems as a function of spin imbalance [18 22 .

For attractive interactions, $E_{\text {pol }}<0$ measures the polaron binding energy in the Fermi sea. In the unitary limit, where the S-wave scattering length $|a| \rightarrow \infty$, the polaron energy is universal at low densities and scales as $E_{\mathrm{pol}}=\eta E_{F}$ where $E_{F}=k_{F}^{2} / 2 m$ is the Fermi energy (with Fermi momentum $k_{F}$ ) and $\eta<0$ is a universal number [18. Neutrons, whose scattering length is large $(a=-18.5 \mathrm{fm})$, have low-density properties close to the unitary limit.

At unitarity, the polaron energy admits a variational bound that sums one-particle-one-hole excitations, $\eta \leq$ -0.6066 [18, which is remarkably close to a full manybody treatment [23, $\eta=-0.6158$, and agrees with Quantum Monte Carlo (QMC) calculations 20 22, 24. These theoretical values are consistent with experimental extractions of $\eta=-0.58(5)$ 25] and $-0.64(7)$ 26] from ultracold atoms across a Feshbach resonance. The Fermi polaron continues to be an exciting area of research [27, 28, with recent studies of the polaron in two dimensions [29, 30] and of the P-wave polaron [31.

Here, we generalize the polaron to strongly interacting neutrons, where the effective range $r_{e}=2.7 \mathrm{fm}$ is important, and $k_{F} r_{e} \sim 1$ is not small, as is relevant for nuclei. We calculate the polaron energy using an effective field theory (EFT) for large $a$ and large $r_{e}$, and from chiral EFT interactions that include contributions beyond the effective range. We benchmark these approximations with $\mathrm{QMC}$ simulations, and compare the resulting $E_{\mathrm{pol}}$ to predictions of nuclear density functionals. Finally, we construct a functional that includes the polaron energy as a constraint.

The Chevy Ansatz [18] for the polaron energy can be generalized to include a large effective range using a di-fermion EFT (dEFT) where the fermions $\psi$ interact through a di-fermion field $d$ and the energy dependence of the di-fermion propagator generates the effective range [3, 32]. The lowest-order dEFT Lagrangian density 
is given by

$$
\begin{aligned}
\mathcal{L}= & \psi^{\dagger}\left(i \partial_{0}+\frac{\nabla^{2}}{2 m}\right) \psi-d^{\dagger}\left(i \partial_{0}+\frac{\nabla^{2}}{4 m}-\Delta\right) d \\
& -g\left(d^{\dagger} \psi \psi+d \psi^{\dagger} \psi^{\dagger}\right)
\end{aligned}
$$

where $\Delta$ and $g$ describe the propagation of the difermion field and its coupling to two fermions, respectively. Matching these to the effective-range expansion with a cutoff regularization (for large cutoffs $\Lambda$ ) gives $\Delta /\left(m g^{2}\right)=1 /(4 \pi a)-\Lambda /\left(2 \pi^{2}\right)$ and $(m / g)^{2}=$ $r_{e} /(8 \pi)-1 /\left(2 \pi^{2} \Lambda\right)$. Upon integrating out the di-fermion field, we obtain an energy-dependent potential between the fermions $V(E)=g^{2} /(\Delta-E)$, where $E$ is the energy in the center-of-mass system. Using $V(E)$ with the Chevy wave function, $|\psi\rangle=\alpha_{0}|\Omega\rangle+\sum_{\mathbf{p}, \mathbf{h}} \alpha_{\mathbf{p}, \mathbf{h}}|\mathbf{p}, \mathbf{h}\rangle$, of spin-up one-particle-one-hole excitations $|\mathbf{p}, \mathbf{h}\rangle\left(p>k_{F}\right.$, $h \leqslant k_{F}$ ) on top of a Fermi sea $|\Omega\rangle$, we find for the polaron energy the self-consistent equation

$$
\begin{aligned}
E_{\mathrm{pol}} & =\int_{0}^{k_{F}} \frac{h^{2} d h}{2 \pi^{2} m}\left[\frac{1}{4 \pi a}-\frac{r_{e}}{8 \pi}\left(m E_{\mathrm{pol}}+\frac{h^{2}}{4}\right)-\frac{k_{F}}{2 \pi^{2}}\right. \\
& \left.-\int_{k_{F}}^{\infty} \frac{p^{2} d p}{(2 \pi)^{2}}\left(\frac{1}{p h} \ln \left|\frac{p^{2}-p h-m E_{\mathrm{pol}}}{p^{2}+p h-m E_{\mathrm{pol}}}\right|+\frac{2}{p^{2}}\right)\right]^{-1} .
\end{aligned}
$$

This is equivalent to the Dyson equation, $E_{\mathrm{pol}}=\Sigma\left(E_{\mathrm{pol}}\right)$, where $\Sigma$ is the self-energy of the spin-down polaron with zero momentum in a Fermi sea of spin-up particles [17. Since the density of spin-down particles vanishes, diagrams involving intermediate spin-down hole states do not exist in the polaron limit. The Chevy ansatz corresponds to calculating $\Sigma$ at the $T$ matrix level, without particle-hole corrections for the single-particle energies, so that at any time in a diagram, there is only one spinup particle-hole excitation from the noninteracting $|\mathbf{p}, \mathbf{h}\rangle$ in the wave function.

The dEFT results for the neutron $E_{\text {pol }}$ as a function of $k_{F}$ are shown in Fig. 1 with and without an effective range. The $r_{e}=0$ result approaches the unitary value $\eta=-0.607$ [18] with increasing $k_{F}$, so that $1 /\left(k_{F} a\right) \rightarrow 0$. For positive $r_{e}$, the neutron polaron binding increases, as observed in Fig. 1 for $k_{F}>0.2 \mathrm{fm}^{-1}$, where $k_{F} r_{e} \approx 0.5$. Intuitively, the polaron interacts with more particles within the range of the interaction. Our results are consistent with Ref. 33, which studied the potential realization of a positive effective range induced by resonant dipolar interactions in ultracold atoms, and [34, 35, which studied narrow resonances. Conversely, for negative $r_{e}$, which is realized with a narrow Feshbach resonance, the polaron binding weakens.

The dEFT makes two approximations: first, it neglects interaction effects beyond the large $a$ and $r_{e}$; second, it is restricted to contributions from one-particle-one-hole excitations. We address the former with microscopic calculations using chiral EFT interactions to next-to-nextto-next-to-leading order $\left(\mathrm{N}^{3} \mathrm{LO}\right)$. Our calculations are



FIG. 1. (Color online) Neutron polaron energy $E_{\text {pol }}$ (in units of the Fermi energy $E_{F}$ ) as a function of Fermi momentum $k_{F}$. The arrows mark the values at unitarity $\eta=-0.607$ and -0.616 (see text). Results are shown from the dEFT calculation with and without an effective range (the $r_{e}=0$ result approaches the unitarity value with increasing $k_{F}$ ) and from QMC calculations with $33+1$ particles and S-wave interactions. The two bands are based on chiral $\mathrm{NN}$ interactions at $\mathrm{N}^{3} \mathrm{LO}$, including also contributions beyond effective-range effects, at the level of third-order particle-particle (pp) ladder contributions (yellow band; 3rd order pp) and resumming pp ladders (red band; pp resummed). The width of the bands reflects the variation from using different cutoffs, different single-particle energies, and for the 3rd order pp band, the difference from second- to third-order ladders.

based on the $500 \mathrm{MeV} \mathrm{N}{ }^{3} \mathrm{LO} \mathrm{NN}$ potential of Ref. 36] and include all partial waves with total angular momentum $J \leqslant 6$. Three-nucleon forces are expected to be small at the densities considered [5, 8]. To study how perturbative the many-body problem is, we also use the renormalization group (RG) 37] to evolve the NN potential to low-momentum interactions $V_{\text {low } k}$ with cutoffs $\Lambda=1.8-2.8 \mathrm{fm}^{-1}$. References [5, 8] show that neutron matter is perturbative at nuclear densities for these interactions, as was recently validated using QMC calculations with chiral EFT interactions [38.

Figure 1 gives our results for the neutron polaron energy based on unevolved and RG-evolved chiral NN interactions at $\mathrm{N}^{3} \mathrm{LO}$. The red band (pp resummed) is obtained at the $T$ matrix level, resumming particle-particle (pp) ladders. The width of the band reflects the variation from using the different cutoffs and either free or Hartree-Fock single-particle energies. The pp resummed results agree with perturbative calculations including up to third-order pp ladder contributions (see Ref. 39] for details), shown by the yellow band (3rd order pp). This latter band also includes an estimate for the perturbative convergence (given by plus/minus the difference from second- to third-order). Similarity RG-evolved interactions lead to analogous results. The perturbative results are shown only for $k_{F} \geqslant 0.5 \mathrm{fm}^{-1}$, because the pp channel becomes nonperturbative at low densities due to the 
large scattering length [37. The microscopic calculations based on chiral NN interactions agree with the dEFT results, indicating that contributions beyond effectiverange effects are small at these densities.

To benchmark the polaron energy and address the previous restriction to one-particle-one-hole excitations, we perform Green's Function Monte Carlo (GFMC) calculations following Refs. 4, 40, 41] with the following Hamiltonian:

$$
\mathcal{H}=-\frac{\hbar^{2}}{2 m} \nabla_{1^{\prime}}^{2}-\frac{\hbar^{2}}{2 m} \sum_{i=1}^{N_{\uparrow}} \nabla_{i}^{2}+\sum_{i=1}^{N_{\uparrow}} V\left(r_{i 1^{\prime}}\right),
$$

where $r_{i 1^{\prime}}$ is the distance between the $i$ 'th majority particle and the impurity $\left(1^{\prime}\right)$. We take the S-wave interaction to be either the ${ }^{1} \mathrm{~S}_{0}$ channel of the Argonne $v_{18}$ neutronneutron potential or a modified Pöschl-Teller potential fit to the scattering length and effective range of the $v_{18}$ potential. Both results agree, demonstrating universality (independence of the potential) over the densities studied with QMC $\left(0.2 \mathrm{fm}^{-1} \leqslant k_{\mathrm{F}} \leqslant 0.7 \mathrm{fm}^{-1}\right)$. Universality is further confirmed by our finding that same-spin $\mathrm{P}$-wave interactions are negligible.

The QMC algorithm evolves an initial state in imaginary time, finding the lowest energy within the space of wave functions having the same nodal structure as the initial state. Since this system has no pairing, we start with a Slater determinant of plane waves for the majority species (as in Ref. 41]), and a single plane wave $e^{i \mathbf{k} \cdot \mathbf{r}_{1^{\prime}}}$ for the impurity. The algorithm thus provides an upper bound for the energy $E_{N_{\uparrow}}$ of the system with $N_{\uparrow}+1$ particles, and therefore to the polaron energy $E_{\mathrm{pol}}=E_{N_{\uparrow}+1}-E_{N_{\uparrow}}$. (Note that these energies are compared at fixed simulation volume $L^{3}=6 \pi^{2} N_{\uparrow} / k_{F}^{3}$, not at fixed total density [42].) Our results for $E_{\text {pol }}$ obtained from $N_{\uparrow}=33$ are presented in Fig. 1.

By varying the momentum of the impurity, we can extract its effective mass by fitting a line to $E_{\text {pol }}(k)$ vs. $k^{2}$ for momenta $(1,2,3,4$ times $2 \pi / L)$ at each density. We find the same increased effective mass $m^{*} / m=1.04(3)$ as in the unitary limit 21] for all densities considered, consistent with microscopic calculations of the Fermi liquid parameters of neutron matter (see, e.g., Ref. [43]).

The QMC results agree with the dEFT and the bands in Fig. 1 for low densities $k_{F} \lesssim 0.5 \mathrm{fm}^{-1}$, but yield a lower energy as the density increases. These differences could be due to nonperturbative many-particle-hole effects neglected by the latter approaches. The QMC calculations are performed in a box, but exhibit very small finite-size effects. The dependence on particle number, shown for the largest density $\left(k_{F}=0.7 \mathrm{fm}^{-1}\right)$ in Fig. 2 , follows the noninteracting system, justifying the use of the $33+1$ particle QMC results to approximate the thermodynamic limit. This finding is consistent with Ref. 4] for the spin-symmetric paired system.

Finally, to assess the impact of tensor and spin-orbit in-

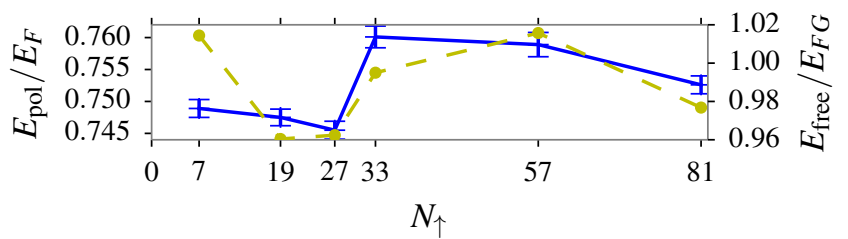

FIG. 2. (Color online) Dependence of the polaron energy $E_{\text {pol }}$ (solid line, left axis, in units of the Fermi energy $E_{F}$ ) on the number of spin-up neutrons $N_{\uparrow}$ for the largest density $\left(k_{F}=0.7 \mathrm{fm}^{-1}\right)$. This follows the dependence of noninteracting neutrons $E_{\text {free }}$ (dashed line, right axis, in units of the Fermi-gas energy $E_{F G}$ ) except for the smallest system $N_{\uparrow}=7$, where the box is only 4 times larger than the effective range.

teractions, we use Auxiliary-Field Diffusion Monte Carlo (AFDMC) [44, 45] to calculate the polaron energy with the Argonne $v_{8}^{\prime}$ potential [46]. We have found that the GFMC algorithm leads to more accurate results for large polarizations, but AFDMC has the advantage that it can include tensor and spin-orbit interactions nonperturbatively. Comparing the AFDMC [vs. GFMC] energies for $33+1$ particles at two intermediate densities we find $E_{\mathrm{pol}} / E_{F}=-0.531 \pm 0.008$ [vs. $-0.522 \pm 0.006$ ] for $k_{F}=0.3 \mathrm{fm}^{-1}$ and $E_{\mathrm{pol}} / E_{F}=-0.567 \pm 0.006$ [vs. $-0.589 \pm 0.005]$ for $k_{F}=0.4 \mathrm{fm}^{-1}$. We have also performed AFDMC simulations at these $k_{F}$ values using the Argonne $v_{6}^{\prime}$ (no spin-orbit) and $v_{4}^{\prime}$ (plus no tensor) interactions, and find consistent results indicating that tensor and spin-orbit interactions have a small effect at these densities. This implies that the polaron lifetime is long, which is consistent with expectations based on calculations of the small spin relaxation rate $\Gamma_{\sigma} \ll E_{\mathrm{pol}} \sim E_{F}$ in neutron matter at low densities [4].

Having established the polaron energy in Fig. 1, we study the impact on nuclear density functionals. We consider the family of Skyrme functionals, which have been used in global studies of nuclei [1. The energy density of neutron matter $\mathcal{E}$ is given by the parametrization [48.

$$
\begin{aligned}
\mathcal{E}= & \frac{\hbar^{2}}{2 m} \tau+\left(C_{0}^{\tau}+C_{1}^{\tau}\right) \rho \tau+\left(C_{0}^{s T}+C_{1}^{s T}\right) \mathbf{s} \cdot \mathbf{T} \\
& +\left(C_{0}^{\rho, 0}+C_{1}^{\rho, 0}\right) \rho^{2}+\left(C_{0}^{\rho, D}+C_{1}^{\rho, D}\right) \rho^{2+\gamma} \\
& +\left(C_{0}^{s, 0}+C_{1}^{s, 0}\right) \mathbf{s}^{2}+\left(C_{0}^{s, D}+C_{1}^{s, D}\right) \mathbf{s}^{2} \rho^{\delta}
\end{aligned}
$$

with density $\rho=\rho_{\uparrow}+\rho_{\downarrow}$, spin density $\mathbf{s}=\rho_{\uparrow}-\rho_{\downarrow}$, kinetic density $\tau=\tau_{\uparrow}+\tau_{\downarrow}$, and spin kinetic density $\mathbf{T}=\tau_{\uparrow}-\tau_{\downarrow}$. The various functionals differ in the set of Skyrme parameters $C$, which follow from fits to selected properties of nuclei and nuclear matter. For neutron matter only the isoscalar plus isovector $\left(C_{0}+C_{1}\right)$ combinations enter. We have allowed the usual density-dependent $C^{D}$ terms to have different powers of the density for the time-even $(\gamma)$ and time-odd $(\delta)$ parts.

The polaron energy follows from the energy density (3) by $E_{\mathrm{pol}}=\left.\left(\partial \mathcal{E} / \partial \rho_{\downarrow}\right)\right|_{\rho_{\downarrow}=0}$. Figure 3 shows the predic- 
tions for $E_{\text {pol }}$ of various state-of-the-art nuclear density functionals: SIII [49], SGII [50], SkM* [51], SLy4 and SLy5 12, SkO and $\mathrm{SkO}^{\prime}$ [52, BSk9 53], SAMi [54, as well as the Gogny D1N functional [13. All functionals predict an attractive polaron energy, but $E_{\text {pol }}$ varies greatly among the different functionals and is generally underestimated. It is apparent that none of the existing functionals can reproduce the universal dependence in the low-density limit. This is expected, because the functionals were not constructed to explore this regime. However, as one approaches nuclear densities the discrepancies persist. Even the SGII, $\mathrm{SkO}^{\prime}$, and $\mathrm{SkM}^{*}$ functionals, which come close to the QMC results around $k_{F} \sim 0.5 \mathrm{fm}^{-1}$, have a stronger density dependence than the microscopic results, and the Gogny D1N functional, which was fit to neutron matter calculations, even differs most from the microscopic $E_{\text {pol }}$ results.

Figure 3 demonstrates that the polaron energy provides a novel constraint for nuclear density functionals. To this end, we construct a new density functional UNEDF1-pol, which we fit to the QMC $E_{\text {pol }}$ results. Because the new exponent $\delta$ appears only in the terms containing s, this allows to fit the QMC results without affecting the time-even part (i.e., the spin-symmetric properties), for which we take the UNEDF1 functional [55]. Because the density dependence of the spin Skyrme parameters $C^{s}$ impacts the spin response of nuclei, we constrain the fit to reproduce the sum of Landau parameters [56] $G_{0}+G_{0}^{\prime}=2.0$, which also avoids possible spin instabilities in nuclear matter. This value was chosen from microscopic calculations of asymmetric nuclear matter [57, because spin resonances are weak, making it difficult to extract $G_{0}$ from experiment. We find $C_{0}^{s, 0}+C_{1}^{s, 0}=48 \pm 24 \mathrm{MeV} \mathrm{fm}^{3}, C_{0}^{s, D}+C_{1}^{s, D}=$ $61 \pm 14 \mathrm{MeV} \mathrm{fm}^{3+\delta}$, and $\delta=-0.288 \pm 0.026$. The large errors on the $C_{0}^{s}+C_{1}^{s}$ can be traced to a high correlation (0.994) with $\delta$, small variations of which significantly change the polaron energy.

In summary, we compute the energy of the neutron polaron, generalizing the polaron of condensed matter physics and ultracold atoms to strong interactions with a significant effective range. We use QMC calculations to benchmark non-perturbative contributions to the polaron energy, finding that for densities $k_{F} \lesssim 0.5 \mathrm{fm}^{-1}$ the polaron energy $E_{\text {pol }}$ and its density dependence agree with results based on a dEFT and with microscopic calculations using chiral EFT interactions at $\mathrm{N}^{3} \mathrm{LO}$ that include contributions beyond the effective range. Our results for the neutron polaron provide an anchor for the equation of state of the neutron-star crust, in particular for the chemical potentials, which are also important for thermal and transport properties (see, e.g., Refs 41, 58).

Finally, we show that current nuclear density functionals do not correctly describe the neutron polaron, and construct a new functional UNEDF1-pol (based on the UNEDF1 55] functional) that satisfies the QMC con-

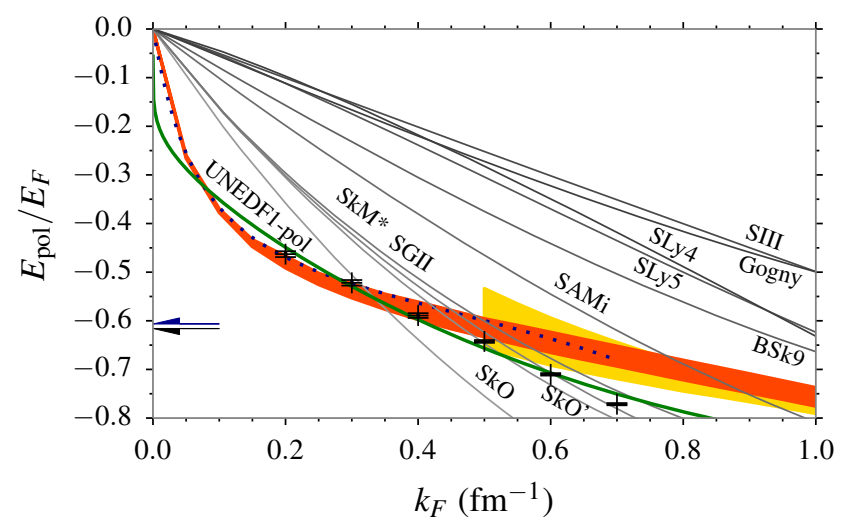

FIG. 3. (Color online) Comparison of the microscopic results for the polaron energy of Fig. 1 with predictions of nuclear density functionals [12, 13, 49,54] (see text). The thick solid (green) curve is the new UNEDF1-pol density functional, which is based on Ref. [55] plus a fit to the QMC $E_{\text {pol }}$ results.

straints by adjusting the time-odd components. These results provide new constraints to guide density functional theory to better describe polarized systems.

We thank A. Bulgac, S. Gandolfi, and P. Massignan for discussions. This work was supported by the Helmholtz Alliance Program of the Helmholtz Association, contract HA216/EMMI "Extremes of Density and Temperature: Cosmic Matter in the Laboratory", the ERC Grant No. 307986 STRONGINT, the Natural Sciences and Engineering Research Council of Canada, the US DOE Grant No. DE-FG02-00ER41132, the NSF Grants No. PHY-0835543, PHY-1002478, and the Polish National Centre for Research and Development within the SARFEN-NUPNET Grant. Computations were carried out at NERSC and at the Jülich Supercomputing Center. We thank the Institute for Nuclear Theory at the University of Washington for its hospitality and the US DOE for partial support.

* E-mail: gezerlis@uoguelph.ca

[1] M. Bender, P.-H. Heenen, and P.-G. Reinhard, Rev. Mod. Phys. 75, 121 (2003).

[2] J. Erler, N. Birge, M. Kortelainen, W. Nazarewicz, E. Olsen, A. M. Perhac, and M. Stoitsov, Nature 486, 509 (2012).

[3] A. Schwenk and C. J. Pethick, Phys. Rev. Lett. 95, 160401 (2005).

[4] A. Gezerlis and J. Carlson, Phys. Rev. C 77, 032801(R) (2008); ibid. 81, 025803 (2010).

[5] K. Hebeler and A. Schwenk, Phys. Rev. C 82, 014314 (2010).

[6] A. Lacour, J. A. Oller, and U.-G. Meißner, Annals Phys. 326, 241 (2011).

[7] S. Gandolfi, J. Carlson, and S. Reddy, Phys. Rev. C 85, 
032801 (2012).

[8] I. Tews, T. Krüger, K. Hebeler, and A. Schwenk, Phys. Rev. Lett. 110, 032504 (2013); T. Krüger, I. Tews, K. Hebeler, and A. Schwenk, Phys. Rev. C 88, 025802 (2013).

[9] J. W. Holt, N. Kaiser, and W. Weise, Prog. Part. Nucl. Phys. 73, 35 (2013).

[10] S. Gandolfi, J. Carlson, and S. C. Pieper, Phys. Rev. Lett. 106, 012501 (2011).

[11] S. A. Fayans, JETP Lett. 68, 169 (1998).

[12] E. Chabanat, P. Bonche, P. Haensel, J. Meyer, and R. Schaeffer, Nucl. Phys. A 635, 231 (1998); ibid. 643, 441(E) (1998).

[13] F. Chappert, M. Girod, and S. Hilaire, Phys. Lett. B 668, 420 (2008).

[14] N. Chamel, S. Goriely, and J. M. Pearson, Nucl. Phys. A 812, 72 (2008).

[15] A. Bulgac, Phys. Rev. A 76, 040502(R) (2007).

[16] F. J. Fattoyev, C. J. Horowitz, J. Piekarewicz, and G. Shen, Phys. Rev. C 82, 055803 (2010); F. J. Fattoyev, W. G. Newton, J. Xu, B.-A. Li, Phys. Rev. C 86, 025804 (2012).

[17] F. Chevy and C. Mora, Rep. Prog. Phys. 73, 112401 (2010).

[18] F. Chevy, Phys. Rev. A 74, 063628 (2006).

[19] A. Bulgac and M. M. Forbes, Phys. Rev. A 75, 031605(R) (2007).

[20] J. Carlson and S. Reddy, Phys. Rev. Lett. 95, 060401 (2005).

[21] C. Lobo, A. Recati, S. Giorgini, and S. Stringari, Phys. Rev. Lett. 97, 200403 (2006).

[22] S. Pilati and S. Giorgini, Phys. Rev. Lett. 100, 030401 (2008).

[23] R. Combescot and S. Giraud, Phys. Rev. Lett. 101, 050404 (2008).

[24] N. Prokof'ev and B. Svistunov, Phys. Rev. B 77, 020408 (2008); ibid. 77, 125101(R) (2008).

[25] Y. Shin, Phys. Rev. A 77, 041603(R) (2008).

[26] A. Schirotzek, C.-H. Wu, A. Sommer, and M. W. Zwierlein, Phys. Rev. Lett. 102, 230402 (2009).

[27] C. Kohstall, M. Zaccanti, M. Jag, A. Trenkwalder, P. Massignan, G. M. Bruun, F. Schreck, and R. Grimm, Nature 485, 615 (2012).

[28] P. Massignan, M. Zaccanti, and G. M. Bruun, Rep. Prog. Phys. 77, 034401 (2014).

[29] S. Zöllner, G. M. Bruun, and C. J. Pethick, Phys. Rev. A 83, 021603(R) (2011); R. Schmidt, T. Enss, V. Pietila, and E. Demler, Phys. Rev. A 85, 021602(R) (2012).

[30] M. Koschorreck, D. Pertot, E. Vogt, B. Fröhlich, M. Feld, and M. Köhl, Nature 485, 619 (2012).

[31] J. Levinsen, P. Massignan, F. Chevy, and C. Lobo, Phys. Rev. Lett. 109, 075302 (2012).

[32] D. B. Kaplan, Nucl. Phys. B 494, 471 (1997); S. R. Beane and M. J. Savage, Nucl. Phys. A 694, 511 (2001).

[33] Z.-Y. Shi, R. Qi, and H. Zhai, Phys. Rev. A 85, 020702(R) (2012).

[34] P. Massignan, Europhys. Lett. 98, 10012 (2012).

[35] C. Trefzger and Y. Castin, Phys. Rev. A 85, 053612 (2012).

[36] D. R. Entem and R. Machleidt, Phys. Rev. C 68, 041001(R) (2003).

[37] S. K. Bogner, R. J. Furnstahl, and A. Schwenk, Prog. Part. Nucl. Phys. 65, 94 (2010).

[38] A. Gezerlis, I. Tews, E. Epelbaum, S. Gandolfi, K. Hebeler, A. Nogga, and A. Schwenk, Phys. Rev. Lett. 111, 032501 (2013).

[39] K. Hebeler, S. K. Bogner, R. J. Furnstahl, A. Nogga, and A. Schwenk, Phys. Rev. C 83, 031301(R) (2011).

[40] A. Gezerlis, Phys. Rev. C 83, 065801 (2011).

[41] A. Gezerlis and R. Sharma, Phys. Rev. C 85, 015806 (2012).

[42] A. Gezerlis, S. Gandolfi, K. E. Schmidt, and J. Carlson, Phys. Rev. Lett. 103, 060403 (2009).

[43] A. Schwenk, B. Friman, and G. E. Brown, Nucl. Phys. A 713, 191 (2003).

[44] K. E. Schmidt and S. Fantoni, Phys. Lett. B 446, 99 (1999).

[45] S. Gandolfi, A. Yu. Illarionov, K. E. Schmidt, F. Pederiva, and S. Fantoni, Phys. Rev. C 79, 054005 (2009).

[46] R. B. Wiringa and S. C. Pieper, Phys. Rev. Lett. 89, 182501 (2002).

[47] S. Bacca, K. Hally, C. J. Pethick, and A. Schwenk, Phys. Rev. C 80, 032802 (2009).

[48] E. Perlińska, S. G. Rohoziński, J. Dobaczewski, and W. Nazarewicz, Phys. Rev. C 69, 014316 (2004).

[49] M. Beiner, H. Flocard, N. Van Giai, and P. Quentin, Nucl. Phys. A 238, 29 (1975).

[50] N. Van Giai and H. Sagawa, Phys. Lett. 106B, 379 (1981).

[51] J. Bartel, P. Quentin, M. Brack, C. Guet, and H.-B. Håkansson, Nucl. Phys. A 386, 79 (1982).

[52] P.-G. Reinhard, D. J. Dean, W. Nazarewicz, J. Dobaczewski, J. A. Maruhn, and M. R. Strayer, Phys. Rev. C 60, 014316 (1999).

[53] S. Goriely, M. Samyn, J. M. Pearson, and M. Onsi, Nucl. Phys. A 750, 425 (2005).

[54] X. Roca-Maza, G. Colò, and H. Sagawa, Phys. Rev. C 86, 031306(R) (2012).

[55] M. Kortelainen, J. McDonnell, W. Nazarewicz, P.-G. Reinhard, J. Sarich, N. Schunck, M. V. Stoitsov, and S. M. Wild, Phys. Rev. C 85, 024304 (2012).

[56] M. Bender, J. Dobaczewski, J. Engel, and W. Nazarewicz, Phys. Rev. C 65, 054322 (2002)

[57] W. Zuo, C. Shen, and U. Lombardo, Phys. Rev. C 67, 037301 (2003)

[58] D. N. Aguilera, V. Cirigliano, J. A. Pons, S. Reddy, and R. Sharma, Phys. Rev. Lett. 102, 091101 (2009). 